\title{
Pembrolizumab (MK-3475) in combination with lenalidomide and low-dose dexamethasone for relapsed/refractory multiple myeloma (RRMM): KEYNOTE-023
}

\author{
Maria-Victoria Mateos ${ }^{1 *}$, David Siegel ${ }^{2}$, Jatin J Shah ${ }^{3}$, Donna Reece ${ }^{4}$, David Avigan ${ }^{5}$, Robert Orlowski ${ }^{6}$, Yang Ge ${ }^{7}$, \\ Arun Balakumaran ${ }^{7}$, Patricia Marinello ${ }^{7}$, Jesus San Miguel ${ }^{8}$ \\ From 30th Annual Meeting and Associated Programs of the Society for Immunotherapy of Cancer (SITC \\ 2015) \\ National Harbor, MD, USA. 4-8 November 2015
}

\section{Background}

The immunomodulatory drug (IMiD) lenalidomide has single-agent activity against RRMM, and synergistic effects when combined with dexamethasone. Overexpression of PD-L1 is associated with tumor invasiveness in MM cells and may be a mechanism of immune evasion. Pembrolizumab, a highly selective, humanized IgG4 anti-PD-1 monoclonal antibody designed to block interaction of PD-1 with PD-L1 and PD-L2, may synergize with IMiDs to enhance tumor suppression. KEYNOTE023 (NCT02036502), an open-label, Phase I, multicenter, nonrandomized, dose-escalation trial will evaluate the safety, tolerability, and efficacy of pembrolizumab in combination with lenalidomide and low-dose dexamethasone in patients with RRMM.

\section{Methods}

Patients $\geq 18$ years with RRMM who have failed $\geq 2$ prior therapies including bortezomib and an IMiD and were refractory to their last line of treatment are eligible. Other key eligibility criteria include measurable disease, ECOG performance status $0 / 1$, and adequate organ function. Key exclusion criteria include history of repeated infections, primary amyloidosis, hyperviscosity, plasma cell leukemia, POEMS syndrome, Waldenström macroglobulinemia, or IgM myeloma; immunosuppressive disorder; prior anti-PD-1/anti-PD-L1 therapy; allogeneic stem cell transplant; or autologous stem cell

${ }^{1}$ Complejo Asistencial Universitario de Salamanca/IBSAL, Salamanca, Spain Full list of author information is available at the end of the article transplant $\leq 12$ weeks from first infusion. Using a modified $3+3$ design followed by toxicity probability interval (TPI) for dose confirmation, cohorts of 3-6 patients per dose level will be enrolled sequentially at escalating doses of pembrolizumab 2, 5, or $10 \mathrm{mg} / \mathrm{kg} \mathrm{Q} 2 \mathrm{~W}$ with low-dose dexamethasone $40 \mathrm{mg}$ and lenalidomide $25 \mathrm{mg}$ until MTD or MAD. Additional patients will receive pembrolizumab in combination with dexamethasone/lenalidomide to confirm the dose based on the TPI algorithm and to evaluate safety and preliminary efficacy. Treatment will continue for 24 months or until complete response, disease progression, or intolerable toxicity. Primary objectives are to establish MTD/ MAD and determine safety and tolerability of the combinations. Efficacy end points are stringent complete response, complete response, and very good partial response rate ( $\mathrm{sCR}+\mathrm{CR}+\mathrm{VGPR}$ rate); ORR; time to response; duration of response; PFS; OS; and correlation of PD-L1 expression with efficacy. Exploratory objectives are PK and relationship between antitumor activity and candidate biomarkers. Response is assessed using International Myeloma Working Group criteria. AEs are graded per NCI CTCAE v4.0 in all patients receiving $\geq 1$ dose. RP2D will be determined from the dose-response relationship and other tolerability data. Descriptive statistics will summarize efficacy end points for each dose level. Recruitment is ongoing.

\section{Trial registration}

ClinicalTrials.gov identifier NCT02036502. 


\section{Authors' details}

'Complejo Asistencial Universitario de Salamanca/IBSAL, Salamanca, Spain. ${ }^{2}$ Hackensack University Medical Center, Hackensack, NJ, USA. ${ }^{3}$ The University of Texas MD Anderson Cancer Center, Houston, TX, USA. ${ }^{4}$ Princess Margaret Cancer Centre, Toronto, ON, Canada. ${ }^{5}$ Beth Israel Deaconess Medical Center, Boston, MA, USA. ${ }^{6}$ The University of Texas MD Anderson Cancer Center, Houston, TX, USA. ${ }^{7}$ Merck \& Co., Inc., Kenilworth, NJ, USA. ${ }^{8}$ Universidad de Navarra, Pamplona, Spain.

Published: 4 November 2015

doi:10.1186/2051-1426-3-S2-P160

Cite this article as: Mateos et al.: Pembrolizumab (MK-3475) in

combination with lenalidomide and low-dose dexamethasone for

relapsed/refractory multiple myeloma (RRMM): KEYNOTE-023. Journal for

ImmunoTherapy of Cancer 2015 3(Suppl 2):P160.

Submit your next manuscript to BioMed Central and take full advantage of:

- Convenient online submission

- Thorough peer review

- No space constraints or color figure charges

- Immediate publication on acceptance

- Inclusion in PubMed, CAS, Scopus and Google Scholar

- Research which is freely available for redistribution

Submit your manuscript at www.biomedcentral.com/submit 